\title{
Asymptotic Solving Essentially Nonlinear Problems
}

\author{
Alexander D. Bruno \\ Department of Singular Problem, Keldysh Institute of Applied Mathematics of RAS, Miusskaya sq. 4, 125047, Moscow, Russia
}

Copyright (C) 2016 by authors, all rights reserved. Authors agree that this article remains permanently

open access under the terms of the Creative Commons Attribution License 4.0 International License

\begin{abstract}
Here we present a way of computation of asymptotic expansions of solutions to algebraic and differential equations and present a survey of some of its applications. The way is based on ideas and algorithms of Power Geometry. Power Geometry has applications in Algebraic Geometry, Differential Algebra, Nonstandard Analysis, Microlocal Analysis, Group Analysis, Tropical/Idempotent Mathematics and so on. We also discuss a connection of Power Geometry with Idempotent Mathematics.
\end{abstract}

Keywords Singularity, Newton Polyhedron, Painlevé Equation, Boundary Layer, Idempotent Analysis

\section{Introduction}

We develop a new Calculus based on Power Geometry [1-4]. At present, it allows to compute local and asymptotic expansions of solutions to nonlinear equations of three classes:

1. algebraic,

2. ordinary differential,

3. partial differential,

as well as to systems of such equations. However, it can also be extended to other classes of nonlinear equations: functional, integral, integro-differential etc.

Principal ideas and algorithms are common for all classes of equations. Computation of asymptotic expansions of solutions consists of 3 following steps (we describe them for one equation $f=0$ ).

1. Calculation of truncated equations $\hat{f}_{j}^{(d)}=0$ by means of generalized faces of the convex polyhedron $\Gamma(f)$ which is a generalization of the Newton polyhedron. The first term of the expansion of a solution to the initial equation $f=0$ is a solution to the corresponding truncated equation $\hat{f}_{j}^{(d)}=0$.

2. Finding solutions to a truncated equation $\hat{f}_{j}^{(d)}=0$, which is quasihomogenous. Using power and logarithmic transformations of coordinates we can reduce the equation $\hat{f}_{j}^{(d)}=0$ to such simple form that can be solved. Among the solutions found we should select appropriate ones which yield the first terms of asymptotic expansions.

3. Computation of the tail of the asymptotic expansion. Each term in the expansion is a solution of a linear equation which can be written down and solved.

Indeed Power Geometry (as a basis of Nonlinear Analysis) can be considered as the third level of Differential Calculus (after Classical Analysis and Functional Analysis). Elements of Plane Power Geometry were proposed by Newton for algebraic equation (1670) [5]; and by Briot and Bouquet for ordinary differential equation of the first order (1856) [6]. Space Power Geometry for a nonlinear autonomous system of Ordinary differential equations (ODEs) was proposed by the author (1962) [1], and for a linear partial differential equation (PDE), by Mikhailov (1963) [7]. Thus, in 2012 we could celebrate 50 years of the first publication on the Newton polyhedron.

Back in the autumn of 1959, I was a third-year student of Department of Mechanics and Mathematics of the Lomonosov Moscow State University, and invented a polyhedron to study asymptotic behavior of solutions to an autonomous system of ODEs near a degenerated stationary point. The polyhedron was described in my work, which was presented at a students' works competition in 1961. In that year Arnold was a postgraduate student, and he became a referee of my work. He estimated my works as not very good, for the mere reason that "geometry of power exponents is useless". In 1962-1970, Arnold wrote reports on some of my articles with the same (rather negative) evaluation of Power Geometry. See details in Section 6 of Chapter 8 of English Edition of my book [3]. However, in 1973 Arnold re-introduced my polyhedron as "Newton polyhedron" and that looked as if he was the inventor of the polyhedron. In fact, he invented only the name [8]. V.P. Maslov was very surprised when I told him (in 1990) that the Newton polyhedron was my invention (he thought that it was Arnold's).

In this paper we intend to explain basic notions of Power Geometry, present some of its algorithms, results, and applications. It is clear that this calculus cannot be mastered using the present paper alone. Power Geometry was the subject of a one-year lecture course "Nonlinear Analysis", taught by the author at the Lomonosov Moscow State University. 


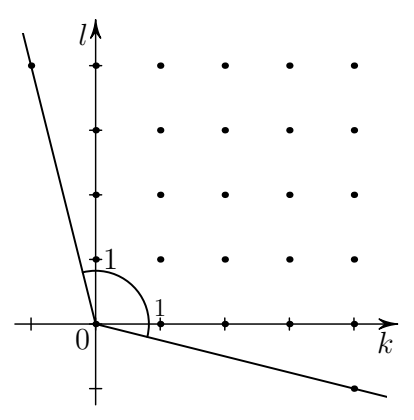

Figure 1. Set of power exponents $k, l$ in expansion of Type 2

\section{Algebraic Equations [2,3]}

We consider a polynomial depending on three variables near its singular point where the polynomial vanishes with all the first partial derivatives. We propose a method of computation of asymptotic expansions of all branches of the set of roots of the polynomial near the above mentioned singular point. There are three types of expansions. The method of computation is based on the spatial Power Geometry. Most of our examples are for polynomials in two variables.

\subsection{The Problem Statement}

Let $X=(x, y, z) \in \mathbb{R}^{3}$ or $\mathbb{C}^{3}$ and $g(X)$ be a polynomial.

Definition 1 A point $X_{0}$ is called singular for the set $\mathcal{G}=$ $\{X: g(X)=0\}$ if all the partial derivatives of the first order of the polynomial $g$ vanish in the point $X_{0}$ and $g\left(X_{0}\right)=0$.

Problem 1 Near the singular point $X_{0}=0$ for each branch of the set $\mathcal{G}$, find a parameter expansion of one of the following three types.

Type 1

$$
x=\sum_{k=1}^{\infty} b_{k} v^{k}, \quad y=\sum_{k=1}^{\infty} c_{k} v^{k}, \quad z=\sum_{k=1}^{\infty} d_{k} v^{k},
$$

where $b_{k}, c_{k}, d_{k}$ are constants.

Type 2

$$
x=\sum b_{k l} u^{k} v^{l}, y=\sum c_{k l} u^{k} v^{l}, z=\sum d_{k l} u^{k} v^{l},
$$

where $b_{k l}, c_{k l}, d_{k l}$ are constants and integer points $(k, l)$ are in a sector with the angle less than $\pi$ (see Fig. 1).

Type 3

$$
x=\sum_{k=0}^{\infty} \beta_{k}(u) v^{k}, y=\sum_{k=0}^{\infty} \gamma_{k}(u) v^{k}, z=\sum_{k=0}^{\infty} \delta_{k}(u) v^{k},
$$

where $\beta_{k}(u), \gamma_{k}(u), \delta_{k}(u)$ are rational functions of $u$ and $\sqrt{\psi(u)}$, and $\psi(u)$ is a polynomial in $u$.

\subsection{Objects and algorithms of Power Geometry}

Consider a finite sum (for example, a polynomial)

$$
g(X)=\sum g_{Q} X^{Q} \text { over } Q \in \mathbf{S}(g),
$$

where $X=(x, y, z) \in \mathbb{C}^{3}, Q=\left(q_{1}, q_{2}, q_{3}\right) \in \mathbb{R}^{3}$ and $X^{Q}=x^{q_{1}} y^{q_{2}} z^{q_{3}}, g_{Q}=$ const $\in \mathbb{C} \backslash\{0\}$. To each of the terms of sum (1), we assign its vector power exponent $Q$, and to the whole sum (1), we assign the set of all vector power exponents of its terms, which is called the support of sum (1) or of the polynomial $g(X)$, and it is denoted by $\mathbf{S}(g)$. The convex hull of the support $\mathbf{S}(g)$ is called the Newton polyhedron of the sum $g(X)$, and it is denoted by $\Gamma(g)$. The boundary $\partial \Gamma$ of the polyhedron $\Gamma(g)$ consists of generalized faces ${ }^{1} \Gamma_{j}^{(d)}$ of various dimensions $d=0,1,2$. Here $j$ is the number of a face. To each generalized face $\Gamma_{j}^{(d)}$, we assign the truncated sum $\hat{g}_{j}^{(d)}(X)=\sum g_{Q} X^{Q}$ over $Q \in \Gamma_{j}^{(d)} \cap \mathbf{S}(g)$.

\section{Example 1: support and the Newton polygon}

We consider the polynomial $g(x, y)=x^{3}+y^{3}-3 x y$. Support $\mathbf{S}(g)$ consists of points $Q_{1}=(3,0), Q_{2}=(0,3)$, $Q_{3}=(1,1)$. The Newton polygon $\Gamma(g)$ is the triangle $Q_{1} Q_{2} Q_{3}$ (Fig. 2). Its edges and corresponding truncated polynomials are

$$
\begin{aligned}
\Gamma_{1}^{(1)}: \hat{g}_{1}^{(1)}=x^{3}-3 x y, \quad & \Gamma_{2}^{(1)}: \hat{g}_{2}^{(1)}=y^{3}-3 x y \\
& \Gamma_{3}^{(1)}: \hat{g}_{3}^{(1)}=x^{3}+y^{3} .
\end{aligned}
$$

Let $\mathbb{R}_{*}^{3}$ be a space dual to the space $\mathbb{R}^{3}$ and $P=$ $\left(p_{1}, p_{2}, p_{3}\right)$ be points of this dual space. The scalar product

$$
\langle Q, P\rangle=q_{1} p_{1}+q_{2} p_{2}+q_{3} p_{3}
$$

is defined for the points $Q \in \mathbb{R}^{3}$ and $P \in \mathbb{R}_{*}^{3}$. In particular, the external normal $N_{k}$ to the generalized face $\Gamma_{k}^{(d)}$ is a point (vector) in $\mathbb{R}_{*}^{3}$.

The scalar product $\left\langle Q, N_{k}\right\rangle$ reaches the maximum value at the points $Q \in \Gamma_{k}^{(d)} \cap \mathbf{S}(g)$, i. e. at the points of the generalized face $\Gamma_{k}^{(d)}$. The set of all points $P \in \mathbb{R}_{*}^{3}$, at which the scalar product (2) reaches the maximum over $Q \in \mathbf{S}(g)$ exactly at points $Q \in \Gamma_{k}^{(d)}$, is called normal cone of the generalized face $\Gamma_{k}^{(d)}$ and is denoted by $\mathbf{U}_{k}^{(d)}$.

\section{Example 2: Normal Cones (cont. of Example 1)}

For faces $\Gamma_{j}^{(d)}$ of the Newton polygon $\Gamma(g)$ of Fig. 2, normal cones are shown in Fig. 3.

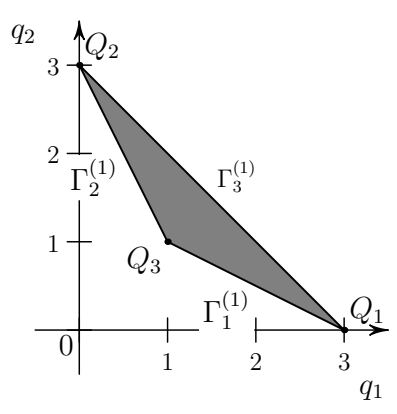

Figure 2. The Newton polygon of polynomial $x^{3}+y^{3}=3 x y$.

For edge $\Gamma_{j}^{(1)}, j=1,2,3$, normal cone $\mathbf{U}_{j}^{(1)}$ is the external ray orthogonal to this edge. For vertex $\Gamma_{j}^{(0)}=Q_{j}$, $j=1,2,3$, normal cone is open sector between external rays orthogonal to edges $\Gamma_{k}^{(1)}$ adjacent to vertex $Q_{j}$.

\footnotetext{
${ }^{1}$ in other terms, facets
} 


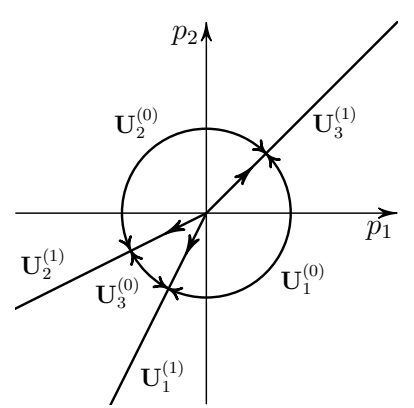

Figure 3. Normal cones $\mathbf{U}_{j}^{(d)}$ for polygon of Fig. 2

Theorem 1 ( [3]) Iffor $t \rightarrow \infty$ the curve

$x=b t^{p_{1}}(1+o(1)), y=c t^{p_{2}}(1+o(1)), z=d t^{p_{3}}(1+o(1))$,

where $b, c, d$ and $p_{i}$ are constants, belongs to the set $\mathcal{G}=$ $\{X: g(X)=0\}$, and the vector $P=\left(p_{1}, p_{2}, p_{3}\right)$ belongs to $\mathbf{U}_{k}^{(d)}$, then the first approximation $x=b t^{p_{1}}, y=c t^{p_{2}}, z=$ $d t^{p_{3}}$ of curve (3) satisfies the truncated equation $\hat{g}_{k}^{(d)}(X)=$ 0 .

The truncated sum $\hat{g}_{j}^{(0)}$ corresponding to the vertex $\Gamma_{j}^{(0)}$ is a monomial. Such truncations are of no interest and will not be considered. We will consider truncated sums corresponding to edges $\Gamma_{j}^{(1)}$ and faces $\Gamma_{j}^{(2)}$ only.

Power transformations are mappings of the form

$$
\log X=B \log X_{1},
$$

where $\log X=(\log x, \log y, \log z)^{\mathrm{T}}, \quad \log X_{1}=$ $\left(\log x_{1}, \log y_{1}, \log z_{1}\right)^{\mathrm{T}}, \quad B$ is a non-degenerate square $3 \times 3$ matrix $\left(b_{i j}\right)$ with rational elements $b_{i j}$ (they are often integer).

The monomial $X^{Q}$ is transformed to the monomial $X_{1}^{Q_{1}}$ by power transformation (4), where $Q_{1}^{\mathrm{T}}=B^{\mathrm{T}} Q^{\mathrm{T}}$. Power transformations and multiplications of polynomial by monomial generate the affine geometry in space $\mathbb{R}^{3}$ of vector power exponents of polynomial monomials. The matrix $B$ with integer elements and $\operatorname{det} B= \pm 1$ is called unimodular.

Theorem 2 ( [3]) For the face $\Gamma_{j}^{(d)}$, there exists a power transformation (4) with an unimodular matrix $B$ which transforms the truncated sum $\hat{g}_{j}^{(d)}(X)$ into the sum in $d$ coordinates, i.e. $\hat{g}_{j}^{(d)}(X)=X_{1}^{Q} h\left(X_{1}\right)$, where $h\left(X_{1}\right)=h\left(x_{1}\right)$ if $d=1$, and $h\left(X_{1}\right)=h\left(x_{1}, y_{1}\right)$ if $d=2$. Here $Q=$ $\left(q_{1}, q_{2}, q_{3}\right) \in \mathbb{R}^{3}$ and other coordinates $y_{1}, z_{1}$ for $d=1$ and $z_{1}$ for $d=2$ are small. If $\hat{g}_{j}^{(d)}(X)$ is a polynomial, then the sum $h\left(X_{1}\right)$ is a polynomial as well.

\subsection{Cone of the problem}

The cone of the problem $\mathbf{L}$ is a convex cone of such vectors

$$
P=\left(p_{1}, p_{2}, p_{3}\right) \in \mathbb{R}_{*}^{3}
$$

that curves of form (3) fill those part of the space $(x, y, z)$ which is under consideration, i. e. must be studied.

So, our initial Problem 1 corresponds to the cone of the problem

$$
\mathbf{L}=\left\{P=\left(p_{1}, p_{2}, p_{3}\right): P<0\right\}
$$

in $\mathbb{R}_{*}^{3}$, since $x, y, z \rightarrow 0$ (and $x, y, z$ as in (3)).

If $x \rightarrow \infty$ then $p_{1}>0$ in the cone of the problem $\mathbf{L}$.

\section{Example 3: Cont. of Examples 1 and 2}

For variables $x, y$ near origin $x=y=0$ cone of the problem is the quadrant III: $\mathbf{L}_{3}=\left\{p_{1}, p_{2}<0\right\}$, near infinity $x=y=\infty$ cone of the problem is the quadrant I: $\mathbf{L}_{1}=\left\{p_{1}, p_{2}>0\right\}$, near point $x=0, y=\infty$ cone of the problem is the quadrant II: $\mathbf{L}_{2}=\left\{p_{1}<0, p_{2}>0\right\}$ (Fig. 4). In Fig. 3 some cones of the problem $\mathbf{L}_{i}$ intersects several normal cones $\mathbf{U}_{j}^{(2)}$. E.g. $\mathbf{L}_{3}$ intersects $\mathbf{U}_{1}^{(1)}, \mathbf{U}_{2}^{(1)}$ and $\mathbf{U}_{1}^{(0)}$, $\mathbf{U}_{2}^{(0)}, \mathbf{U}_{3}^{(0)} . \mathbf{L}_{1}$ intersects $\mathbf{U}_{3}^{(1)}, \mathbf{U}_{1}^{(0)}, \mathbf{U}_{2}^{(0)}$.

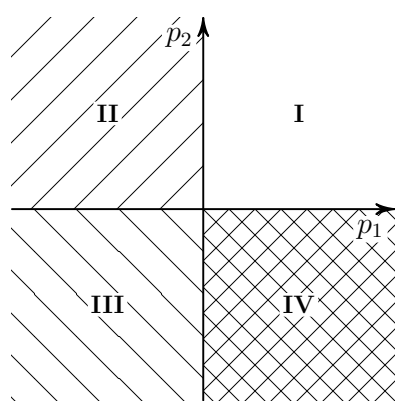

Figure 4. Cones of the problem.

\subsection{Steps for Problem solving}

Step 1. We compute the support $\mathbf{S}(g)$, the Newton polyhedron $\Gamma(g)$, its two-dimensional faces $\Gamma_{j}^{(2)}$ and their external normals $N_{j}$. Using normals $N_{j}$ we compute the normal cones $\mathbf{U}_{k}^{(1)}$ to edges $\Gamma_{k}^{(1)}$.

Step 2. We find all the edges $\Gamma_{k}^{(1)}$ and faces $\Gamma_{j}^{(2)}$, whose normal cones intersect the cone of the problem $\mathbf{L}$. It is enough to select all the faces $\Gamma_{j}^{(2)}$, whose external normals $N_{j}$ intersect the cone of the problem $\mathbf{L}$, and then add all the edges $\Gamma_{k}^{(1)}$ of these faces.

Step 3.

- For each of the selected edges $\Gamma_{k}^{(1)}$, we perform a power transformation $X \rightarrow X_{1}$ of Theorem 2 and we get the truncated equation in a form $h\left(x_{1}\right)=0$.

- We find the roots of this equation. Let $x_{1}^{0}$ be one of its roots.

- We perform the power transformation $X \rightarrow X_{1}$ in the whole polynomial $g(X)$ and we get the polynomial $g_{1}\left(X_{1}\right)=g(X)$.

- We make the shift of variables $x_{2}=x_{1}-x_{1}^{0}, y_{2}=$ $y_{1}, z_{2}=z_{1}$ in the polynomial $g_{1}(X)$ and get the polynomial $g_{2}\left(X_{2}\right)=g_{1}\left(X_{1}\right)$.

- If $x_{1}^{0}$ is a simple root of the equation $h\left(x_{1}\right)=0$ then, according to the Implicit Function Theorem, it corresponds to an expansion of the form $x_{2}=$ $\sum a_{k l} y_{1}^{k} z_{1}^{l}$, where $a_{k l}$ are constants. It gives an expansion of type 2 in coordinates $X$.

- If $x_{1}^{0}$ is a multiple root of the equation $h\left(x_{1}\right)=0$ then we compute the Newton polyhedron of the polynomial $g_{2}\left(X_{2}\right)$, compute the new cone of the problem $\mathbf{L}_{2}$ as the convex cone generated by vector $(-1,0,0)$ and two external normals of faces adjacent the edge, and we continue as above and as follows. 
Step 4.

- For each of the selected faces $\Gamma_{j}^{(2)}$, we perform a power transformation $X \rightarrow X_{1}$ of Theorem 2 and we get a truncated equation in the form $h\left(x_{1}, y_{1}\right)=0$.

- We factorize $h\left(x_{1}, y_{1}\right)$ into prime factors. Let $\tilde{h}\left(x_{1}, y_{1}\right)$ be one of such factors and its algebraic curve has genus $\rho$.

- If $\rho=0$ then there exists birational uniformization $x_{1}=\xi\left(y_{2}\right), y_{1}=\eta\left(y_{2}\right)$ of this curve. We change variables $x_{1}=\xi\left(y_{2}\right)+x_{2}, y_{1}=\eta\left(y_{2}\right)$ and then $\tilde{h}$ is divided by $x_{2}$. We change variables in the whole polynomial $g(X)$ and get the polynomial $g_{2}\left(X_{2}\right) \stackrel{\text { def }}{=} g_{1}\left(X_{1}\right)=g(X)$.

- If $\tilde{h}\left(x_{1}, y_{1}\right)$ is simple factor of $h\left(x_{1}, y_{1}\right)$ then roots of the polynomial $g_{2}\left(X_{2}\right)$ are expanded into series of the form

$$
x_{2}=\sum_{k=1}^{\infty} \alpha_{k}\left(y_{2}\right) z_{2}^{k},
$$

where $\alpha_{k}\left(y_{2}\right)$ are rational functions of $y_{2}$. It gives an expansion of type 3 in original coordinates $X$.

- If $\tilde{h}\left(x_{1}, y_{1}\right)$ is a multiple factor of $h\left(x_{1}, y_{1}\right)$ then we compute the Newton polyhedron of the polynomial $g_{2}\left(X_{2}\right)$, compute the cone of the problem $\mathbf{L}_{2}=\left\{P: p_{2}, p_{3}<0\right\}$ and continue computations.

- If $\rho=1$ (elliptic curve), there exists the birational change of variables $x_{1}, y_{1} \rightarrow x_{2}, y_{2}$ transforming $\tilde{h}\left(x_{1}, y_{1}\right)$ into the normal form $x_{2}^{2}-\psi\left(y_{2}\right)$, where $\psi$ is a polynomial of order 3 or 4 .

- If $\rho>1$, we distinguish hyper-elliptic and non hyper-elliptic curves. The hyper-elliptic curve is birationally equivalent $x_{1}, y_{1} \rightarrow x_{2}, y_{2}$ to its normal form $x_{2}^{2}-\psi\left(y_{2}\right)$, where $\psi$ is a polynomial of order $2 \rho+1$ or $2 \rho+2$.

- If $\rho \geqslant 1$ and we have the (hyper)elliptic curve and factor $\tilde{h}$ of $h$ is simple we get expansions of solutions of equation $g_{2}\left(X_{2}\right)=0$ into series (5), where $\alpha_{k}$ are rational functions of $y_{2}$ and $\sqrt{\psi\left(y_{2}\right)}$. We get the expansion of type 3 in original coordinates $X$.

- If $\rho \geqslant 1$ and we have the (hyper)elliptic curve and $\tilde{h}\left(x_{1}, y_{1}\right)$ is a multiple factor of $h\left(x_{1}, y_{1}\right)$ then we continue for $g_{2}\left(X_{2}\right)$ as above.

In this procedure we distinguish two cases:

Case 1. Truncated polynomial contains linear part of one of the variables or $x_{1}^{0}$ is a simple root of $h\left(x_{1}\right)$ or $\tilde{h}\left(x_{1}, y_{1}\right)$ is simple factor of $h\left(x_{1}, y_{1}\right)$. Then a generalization of Implicit Function Theorem is applicable and it is possible to compute parametric expansion of set of roots of full polynomial.

Case 2. Truncated polynomial does not contain linear part of any variable and $x_{1}^{0}$ is a multiple root of $h\left(x_{1}\right)$ or $\tilde{h}\left(x_{1}, y_{1}\right)$ is a multiple factor of $h\left(x_{1}, y_{1}\right)$. Then the Newton polyhedron for full polynomial must be built and we must consider new truncated polynomials taking into account the new cone of the problem $\mathbf{L}$.

\section{Example 4 (cont. of Examples 1-3)}

- For edge $\Gamma_{1}^{(1)}$, we get truncated equation $x^{2}-3 y=0$, i. e. $y=x^{2} / 3$. It is the case 1 , and this asymptotic form is continued into power expansion of branch $y=$ $x^{2} / 3+\sum_{k=2}^{\infty} b_{k} x^{2 k}$ near the origin $x=y=0$.

- For edge $\Gamma_{2}^{(1)}$, we get truncated equation $y^{2}-3 x=0$, i. e. $y= \pm \sqrt{3 x}$. It is the case 1 , and these asymptotic forms are continued into power expansions of branches $y= \pm \sqrt{3 x}+\sum_{k=2}^{\infty} b_{k} x^{k / 2}$ near the origin $x=y=0$.

- For edge $\Gamma_{3}^{(1)}$, we get truncated equation $x^{3}+y^{3}=0$. It has the simple factor $x+y=0$, i. e. $y=-x$. It is again case 1 of simple root, and the power expansion at infinity $y=-x-1+\sum_{k=1}^{\infty} b_{k} x^{-k}$ gives the asymptote $x+y=-1$ for the curve $g(x, y)=0$ called folium of Descartes (Fig. 5).

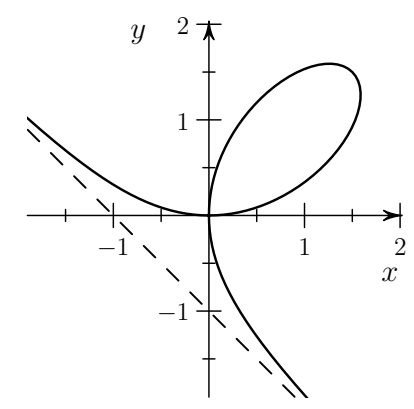

Figure 5. Folium of Descartes

Asymptotic description of a subset of singular points of $\mathcal{G}$ can be obtained by the same procedure, but we have to select only singular points in each truncated equation. As result we obtain expansions of type 1 .

\subsection{Results}

Theorem 3 ( [4]) The algorithm of Subsection 2.4, in the case when all curves formed by roots of corresponding twodimensional truncated equations with positive genus are elliptic or hyper-elliptic, yields a local description of all components of the set $\mathcal{G}$, adjacent to the starting point $X_{0}$, in form of expansions of types 1-3.

\subsection{Implementation and Application}

Implementation of the described algorithm see in [9]. Its application to computation of a set of stability of a certain ODE system depending on several parameters see in [10]. 


\section{Example 5 [9]}

$$
\begin{aligned}
g(X)= & 512 z^{6}-4352 z^{5} y-768 z^{5} x+14848 z^{4} y^{2} \\
& +5376 z^{4} y x+512 z^{4} x^{2}-25408 z^{3} y^{3}-14656 z^{3} y^{2} x \\
& -2752 z^{3} y x^{2}-192 z^{3} x^{3}+21800 z^{2} y^{4} \\
& +19168 z^{2} y^{3} x+5360 z^{2} y^{2} x^{2}+736 z^{2} y x^{3}+40 z^{2} x^{4} \\
& -7500 z y^{5}-11700 z y^{4} x-4376 z y^{3} x^{2}-904 z y^{2} x^{3} \\
& -92 z y x^{4}-4 z x^{5}+2500 y^{5} x+1200 y^{4} x^{2} \\
& +344 y^{3} x^{3}+48 y^{2} x^{4}+4 y x^{5}-256 z^{5}+2880 z^{4} y \\
& +1344 z^{4} x-14976 z^{3} y^{2}-6720 z^{3} y x-1344 z^{3} x^{2} \\
& +37928 z^{2} y^{3}+13816 z^{2} y^{2} x+5144 z^{2} y x^{2} \\
& +456 z^{2} x^{3}-45120 z y^{4}-14464 z y^{3} x-6784 z y^{2} x^{2} \\
& -1152 z y x^{3}-64 z x^{4}+20250 y^{5}+6490 y^{4} x \\
& +3156 y^{3} x^{2}+740 y^{2} x^{3}+82 y x^{4}+2 x^{5}+1872 z^{4} \\
& +2016 z^{3} y-5088 z^{3} x-35496 z^{2} y^{2}+15888 z^{2} y x \\
& +2200 z^{2} x^{2}+67608 z y^{3}-12936 z y^{2} x-5176 z y x^{2} \\
& -344 z x^{3}-37827 y^{4}+828 y^{3} x+2782 y^{2} x^{2} \\
& +412 y x^{3}+13 x^{4}-13824 z^{3}+62208 z^{2} y \\
& +6912 z^{2} x-93312 z y^{2}-20736 z y x-1152 z x^{2} \\
& +46656 y^{3}+15552 y^{2} x+1728 y x^{2}+64 x^{3} .
\end{aligned}
$$

The structure of solutions of the algebraic equation $g(X)=0$ near its singular points (including infinity). The Newton polyhedron of this equation is shown on Fig. 6 .

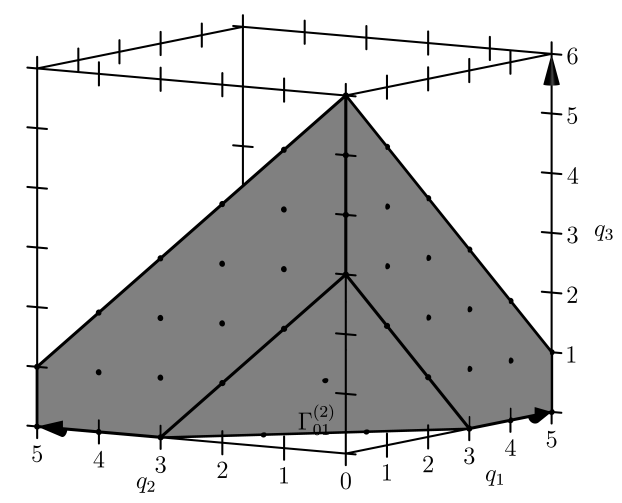

Figure 6. The Newton polyhedron for polynomial $g(X)$.

Near origin $X=0$ we obtain

$x=\sum_{k=0}^{\infty} \beta_{k}(u) v^{k}, \quad y=\sum_{k=0}^{\infty} \gamma_{k}(u) v^{k}, \quad z=\sum_{k=0}^{\infty} \delta_{k}(u) v^{k}$,

where $\beta_{k}(u), \gamma_{k}(u), \delta_{k}(u)$ are rational functions of $u$. More precisely, we have:

$$
\begin{aligned}
& x=\Omega_{1}(u)\left(12 v^{3}+18 \Omega_{2}(u) \frac{27 u^{2}+10 u-5}{u+1} v^{4}\right)+o\left(v^{4}\right), \\
& y=\Omega_{1}(u)\left(2 v^{2}+12 \Omega_{2}(u)(5 u-1) v^{3}\right)+o\left(v^{3}\right) \\
& z=\Omega_{1}(u)\left(3 v^{2}+4 \Omega_{2}(u) \frac{71 u^{2}+13 u-4}{3 u+1} v^{3}\right)+o\left(v^{3}\right)
\end{aligned}
$$

here $\Omega_{1}(u)=\frac{54(3 u+1)^{3}}{(7 u+1)(u+1)^{2}}, \quad \Omega_{2}(u)=$ $\frac{3 u+1}{(7 u+1)(u+1)}$.

\section{Ordinary Differential Equations. Algebraic Approach}

\subsection{Plane Power Geometry [11]}

First, consider one differential equation and power expansions of its solutions (later we consider more complicated expansions).

Let $x$ be independent and $y$ be dependent variables, $x, y \in$ $\mathbb{C}$. A differential monomial $a(x, y)$ is a product of an ordinary monomial $c x^{q_{1}} y^{q_{2}}$, where $c=$ const $\in \mathbb{C},\left(q_{1}, q_{2}\right) \in \mathbb{R}^{2}$, and a finite number of derivatives of the form $d^{l} y / d x^{l}, l \in \mathbb{N}$. A sum of differential monomials

$$
f(x, y)=\sum a_{i}(x, y)
$$

is called the differential sum.

Problem 2 Let a differential equation be given

$$
f(x, y)=0
$$

where $f(x, y)$ is a differential sum. As $x \rightarrow 0$, or as $x \rightarrow \infty$, for solutions $y=\varphi(x)$ to equation (7), find all expansions of the form

$$
y=c_{r} x^{r}+\sum c_{s} x^{s}, \quad c_{r}=\text { const } \in \mathbb{C}, \quad c_{r} \neq 0,
$$

where $c_{s}$ are polynomials in $\log x$, and power exponents $r, s \in \mathbb{R}, \omega r>\omega s$, and $\omega=-1$, if $x \rightarrow 0$, or $\omega=1$, if $x \rightarrow$ $\infty$.

The procedure to compute expansions (8) consists of two steps: computation of the first approximations $y=c_{r} x^{r}$, $c_{r} \neq 0$ and computation of further expansion terms in (8).

To each differential monomial $a(x, y)$, we assign its (vector) power exponent $Q(a)=\left(q_{1}, q_{2}\right) \in \mathbb{R}^{2}$ by the following rules:

$$
Q\left(c x^{q_{1}} y^{q_{2}}\right)=\left(q_{1}, q_{2}\right) ; \quad Q\left(d^{l} y / d x^{l}\right)=(-l, 1)
$$

when differential monomials are multiplied, their power exponents must be added as vectors $Q\left(a_{1} a_{2}\right)=Q\left(a_{1}\right)+Q\left(a_{2}\right)$. The set $\mathbf{S}(f)$ of power exponents $Q\left(a_{i}\right)$ of all differential monomials $a_{i}(x, y)$ presented in differential sum (6) is called the support of the sum $f(x, y)$.

Obviously, $\mathbf{S}(f) \in \mathbb{R}^{2}$. The convex hull $\Gamma(f)$ of the support $\mathbf{S}(f)$ is called the polygon of the sum $f(x, y)$. The boundary $\partial \Gamma(f)$ of the polygon $\Gamma(f)$ consists of the vertices $\Gamma_{j}^{(0)}$ and the edges $\Gamma_{j}^{(1)}$. They are called (generalized) faces $\Gamma_{j}^{(d)}$, where the upper index indicates the dimension of the face, and the lower one is its number. Each face $\Gamma_{j}^{(d)}$ corresponds to the truncated sum

$$
\hat{f}_{j}^{(d)}(x, y)=\sum a_{i}(x, y) \text { over } i: Q\left(a_{i}\right) \in \Gamma_{j}^{(d)} \cap \mathbf{S}(f)
$$

and to truncated equation $\hat{f}_{j}^{(d)}(x, y)=0$ of equation (7).

\section{Example 6}

Consider the third Painlevé equation $f(x, y) \stackrel{\text { def }}{=}-x y y^{\prime \prime}+x y^{\prime 2}-y y^{\prime}+a y^{3}+b y+c x y^{4}+d x=0$, 


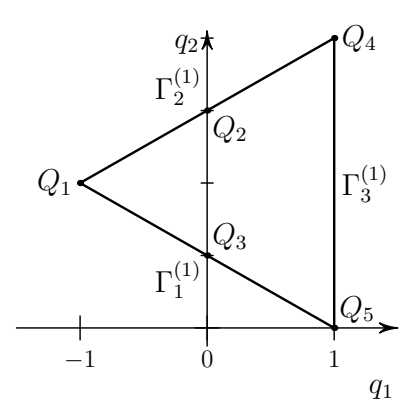

Figure 7. The Newton polygon of equation (10)

assuming the complex parameters $a, b, c, d \neq 0$. Here the first three differential monomials have the same power exponent $Q_{1}=(-1,2)$, then $Q_{2}=(0,3), Q_{3}=(0,1)$, $Q_{4}=(1,4), Q_{5}=(1,0)$. They are shown in Fig. 7 in coordinates $q_{1}, q_{2}$.

Their convex hull $\Gamma(f)$ is the triangle with three vertices $\Gamma_{1}^{(0)}=Q_{1}, \Gamma_{2}^{(0)}=Q_{4}, \Gamma_{3}^{(0)}=Q_{5}$, and with three edges $\Gamma_{1}^{(1)}, \Gamma_{2}^{(1)}, \Gamma_{3}^{(1)}$. The vertex $\Gamma_{1}^{(0)}=Q_{1}$ corresponds to the truncation

$$
\hat{f}_{1}^{(0)}(x, y)=-x y y^{\prime \prime}+x y^{\prime 2}-y y^{\prime},
$$

and the edge $\Gamma_{1}^{(1)}$ corresponds to the truncation

$$
\hat{f}_{1}^{(1)}(x, y)=\hat{f}_{1}^{(0)}(x, y)+b y+d x .
$$

Let the plane $\mathbb{R}_{*}^{2}$ be dual to the plane $\mathbb{R}^{2}$ such that for $P=$ $\left(p_{1}, p_{2}\right) \in \mathbb{R}_{*}^{2}$ and $Q=\left(q_{1}, q_{2}\right) \in \mathbb{R}^{2}$, the scalar product

$$
\langle P, Q\rangle \stackrel{\text { def }}{=} p_{1} q_{1}+p_{2} q_{2}
$$

is defined. Each face $\Gamma_{j}^{(d)}$ corresponds to its own normal cone $\mathbf{U}_{j}^{(d)} \subset \mathbb{R}_{*}^{2}$ formed by the external normal vectors $P$ to the face $\Gamma_{j}^{(d)}$. For the edge $\Gamma_{j}^{(1)}$, the normal cone $\mathbf{U}_{j}^{(1)}$ is the ray orthogonal to the edge $\Gamma_{j}^{(1)}$ and directed outward the polygon $\Gamma(f)$. For the vertex $\Gamma_{j}^{(0)}$, the normal cone $\mathbf{U}_{j}^{(0)}$ is the open sector (angle) in the plane $\mathbb{R}_{*}^{2}$ with the vertex at the origin $P=0$ and limited by the rays which are the normal cones of the edges adjacent to the vertex $\Gamma_{j}^{(0)}$.

\section{Example 7 (cont. of Example 6)}

For equation (10), the normal cones $\mathbf{U}_{j}^{(d)}$ of the faces $\Gamma_{j}^{(d)}$ are shown in Fig. 8.

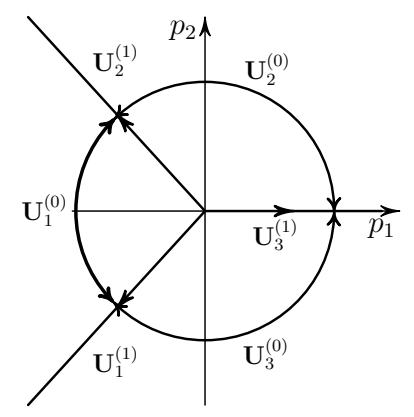

Figure 8. The normal cones $\mathbf{U}_{j}^{(d)}$ for polygon of Fig. 7

Thus, each face $\Gamma_{j}^{(d)}$ corresponds to the normal cone $\mathbf{U}_{j}^{(d)}$ in the plane $\mathbb{R}_{*}^{2}$ and to the truncated equation $\hat{f}_{j}^{(d)}(x, y)=0$.
Theorem 4 ( [11]) If expansion (8) satisfies equation (7), and $\omega(1, r) \in \mathbf{U}_{j}^{(d)}$, then the truncation $y=c_{r} x^{r}$ of solution (8) is the solution to truncated equation (9).

As truncated equation is quasi-homogeneous it is not difficult to find its power solutions. Hence, to find all truncated solutions $y=c_{r} x^{r}$ to equation (7), we need to compute: the support $\mathbf{S}(f)$, the polygon $\Gamma(f)$, all its faces $\Gamma_{j}^{(d)}$, and their normal cones $\mathbf{U}_{j}^{(d)}$. Then for each truncated equation $\hat{f}_{j}^{(d)}(x, y)=0$, we need to find all its solutions $y=c_{r} x^{r}$ which have one of the vectors $\pm(1, r)$ lying in the normal cone $\mathbf{U}_{j}^{(d)}$.

For each power solution $y=c_{r} x^{r}$ of the truncated equation $\hat{f}_{j}^{(d)}=0$, we can compute its characteristic polynomial $\nu(k)$. Roots $k_{j}$ of $\nu(k)$ with $\omega k_{j}<\omega r$ are critical numbers of the solution $c_{r} x^{r}$.

\section{Example 8 (cont. of Examples 6, 7)}

For the truncated equation $\hat{f}_{1}^{(0)}=0$ with $\omega=-1$, we have solutions $y=c_{r} x^{r}$ with arbitrary constants $r$ and $c_{r}$. But according to Theorem 4, $y=c_{r} x^{r}$ can be a formal asymptotic form of a solution to full equation (7) if $\omega(1, r) \subset \mathbf{U}_{1}^{(0)}$, i. e. $-1<r<1$. Corresponding characteristic polynomial $\nu(k)=-(k-r)^{2}$. Hence, here we have no critical numbers.

For the truncated equation $\hat{f}_{1}^{(1)}=0$, we have power solution $y=-d x / b$. Its characteristic polynomial is $\nu(k)=$ $(k-1)^{2}+b^{2} / d$.

Using support $\mathbf{S}(f)$ of the equation (7) and critical numbers $k_{1}, \ldots, k_{\varkappa}$ with $\omega r>\omega k_{i}$, we can find the set $\mathbf{K}\left(k_{1}, \ldots, k_{\varkappa}\right) \subset \mathbb{R}$, support of expansion (8). Its elements $s$ satisfy the inequality $\omega r>\omega s$.

Theorem 5 ( [11]) If $y=c_{r} x^{r}, c=$ const, $\omega(1, r) \in \mathbf{U}_{j}^{(d)}$, is a power solution to truncated equation (9), then equation (7) has an expansion of solutions of the form

$$
z=c_{r} x^{r}+\sum c_{s} x^{s} \quad \text { over } \quad s \in \mathbf{K}\left(k_{1}, \ldots, k_{\varkappa}\right),
$$

where $k_{1}, \ldots, k_{\varkappa}$ are critical numbers of the truncated solution $y=c_{r} x^{r} ; c_{s}$ are polynomials in $\log x$, which are uniquely determined for $s \neq k_{i}$. If all critical numbers $k_{1}, \ldots, k_{\varkappa}$ are simple roots, and each $k_{i}$ does not lie in the set $\mathbf{K}\left(k_{1}, \ldots, k_{i-1}, k_{i+1}, \ldots, k_{\varkappa}\right)$, then all coefficients $c_{s}$ are constant; for $s \neq k_{i}$, they are uniquely determined; and for $s=k_{i}$, they are arbitrary.

\section{Example 9 (cont. of Examples 6-8)}

For the truncated solution $y=c x^{r}$ to $\hat{f}_{1}^{(0)}=0$, arbitrary $c \neq 0, r \in(-1,1)$

$\mathbf{K}=\{s=r+l(1-r)+m(1+r), l, m \in \mathbb{N} \cup\{0\}, l+m>0\}$.

Since there are no critical numbers, then all $c_{s}$ are constant and uniquely determined in expansion (12).

For the truncated solution $y=-d x / b$ to $\hat{f}_{1}^{(1)}=0$, we have $\mathbf{K}=\{s=1+2 l$, integral $l>0\}$. If $\Im(b / \sqrt{-d}) \neq 0$, then there are no real critical numbers, and all power exponents $s$ are odd integers greater than 1 in expansion (12), and coefficients $c_{s}$ are constant and uniquely determined. If 
$\Im(b / \sqrt{-d})=0$, then there is a unique real critical number $k_{1}=1+|b / \sqrt{-d}|$, and

$\mathbf{K}\left(k_{1}\right)=\left\{s=1+2 l+m\left(k_{1}-1\right), l, m \in \mathbb{N} \cup\{0\}, l+m>0\right\}$.

Hence, if the number $k_{1}$ is not odd, then all $c_{s}$ are constant and uniquely determined in expansion (12) for $s \neq k_{1}$, and $c_{k_{1}}$ is arbitrary. Finally, if $k_{1}$ is odd, then $\mathbf{K}\left(k_{1}\right)=\mathbf{K}$, and in expansion (12) $c_{s}$ is a uniquely determined constant if $s<$ $k_{1} ; c_{k_{1}}$ is a linear function of $\log x$ with an arbitrary constant term; $c_{s}$ is a uniquely determined polynomial in $\log x$ if $s>$ $k_{1}$.

The truncated equation $\hat{f}_{j}^{(d)}(x, y)=0$ can have non-power solutions $y=\varphi(x)$ which are the asymptotic forms for solutions to the initial equation $f(x, y)=0$. These non-power solutions $y=\varphi(x)$ may be found using power and logarithmic transformations. Power transformation is linear in logarithms and defined by

$$
\begin{gathered}
\log x=b_{11} \log u+b_{12} \log v, \\
\log y=b_{21} \log u+b_{22} \log v, \\
B=\left(\begin{array}{ll}
b_{11} & b_{12} \\
b_{21} & b_{22}
\end{array}\right), b_{i j} \in \mathbb{R}, \operatorname{det} B \neq 0 .
\end{gathered}
$$

It induces linear dual transformations in spaces $\mathbb{R}^{2}$ and $\mathbb{R}_{*}^{2}$. Logarithmic transformation has the forms

$$
\xi=\log u \text { or } \eta=\log v .
$$

\section{Example 10 (cont. of Examples 6-9)}

For the truncated equation (11) corresponding to the edge $\Gamma_{1}^{(1)}$ with the normal vector $-(1,1)$, we make power transformation

$$
\begin{aligned}
\log x & =\log u, \\
\log y & =\log u+\log v, \\
B & =\left(\begin{array}{ll}
1 & 0 \\
1 & 1
\end{array}\right),
\end{aligned}
$$

i. e. $x=u, y=u v$. Since $y^{\prime}=x v^{\prime}+v, y^{\prime \prime}=x v^{\prime \prime}+2 v^{\prime}$, then, canceling $x$ and collecting similar terms, the equation (11), becomes

$$
-x^{2} v v^{\prime \prime}+x^{2} v^{\prime 2}-x v v^{\prime}+b v+d=0 .
$$

Its support consists of three points $\widetilde{Q}_{1}=(0,2), \widetilde{Q}_{2}=(0,1)$, $\widetilde{Q}_{3}=0$ on the axis $\tilde{q}_{1}=0$ (see Fig. 9).

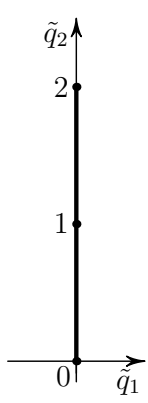

Figure 9. The support and Newton polygon for the third Painlevé equation (15)

Now we make the logarithmic transformation $\xi=\log x$. Since $v^{\prime}=\dot{v} / x, v^{\prime \prime}=(\ddot{v}-\dot{v}) / x^{2}$, where ${ }^{\cdot}=d / d \xi$,

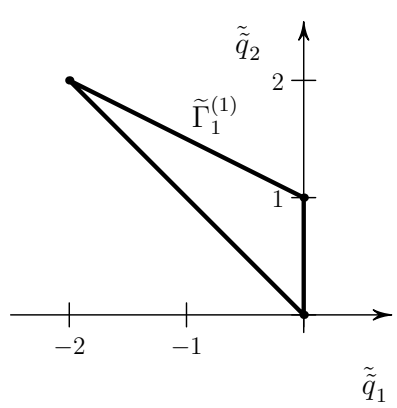

Figure 10. Support and Newton polygon of equation $-v \ddot{v}+\dot{v}^{2}+b v+d=0$.

then, collecting similar terms, the equation (15) takes the form $-v \ddot{v}+\dot{v}^{2}+b v+d=0$. Its support and polygon are shown in Fig. 10. Applying the technique described before to this equation, we obtain the expansion of its solutions $v=$ $-(b / 2) \xi^{2}+\tilde{c} \xi+\sum_{k=0}^{\infty} c_{k} \xi^{-k}$, where $\tilde{c}$ is an arbitrary constant, and the constants $c_{k}$ are uniquely determined. In original variables, we obtain the family of non-power asymptotic forms $y \sim x\left[-(b / 2)(\log x)^{2}+\tilde{c} \log x+\sum_{k=0}^{\infty} c_{k}(\log x)^{-k}\right]$ of solutions to the initial equation (10), when $x \rightarrow 0$.

\subsection{Complex power exponents [11]}

Indeed, the described method allows to calculate solutions with complex power exponents as well.

Thus, by the algebraic approach, expansions of solutions

$$
y=c_{r} x^{r}+\sum_{s} c_{s} x^{s}
$$

with complex power exponents $r$ and $s$, where $\omega \Re r \geqslant \omega \Re s$, and coefficients $c_{r}$ and $c_{s}$ are power series in $\log x, \log \log x$ and so on, are found in a similar way.

In classical analysis, we encounter expansions in fractional powers and with constant coefficients, but here we obtain more complicated expansions of solutions.

\subsection{Algorithms of Power Geometry}

1. Computation of truncated equations and accompanying objects.

2. Solution of truncated equations.

3. Power transformations.

4. Logarithmic transformations.

5. Introducing independent variable $x^{i}$ instead of $x$.

6. Computation of the first variation of a sum.

7. Computation of expansions of solutions to the initial equation, beginning by solutions to a truncated equation.

All these algorithms, except for 4 and 5, can be applied to solve algebraic equations.

Similar technique is used for equations having small or big parameters. The power exponents of these parameters are accounted in the same way as power exponents of variables tending to zero or infinity. 


\subsection{The sixth Painlevé equation [12]}

It has the form

$$
\begin{array}{r}
y^{\prime \prime}=\frac{\left(y^{\prime}\right)^{2}}{2}\left(\frac{1}{y}+\frac{1}{y-1}+\frac{1}{y-x}\right) \\
-y^{\prime}\left(\frac{1}{x}+\frac{1}{x-1}+\frac{1}{y-x}\right)+\frac{y(y-1)(y-x)}{x^{2}(x-1)^{2}}[a \\
\left.+b \frac{x}{y^{2}}+c \frac{x-1}{(y-1)^{2}}+d \frac{x(x-1)}{(y-x)^{2}}\right],
\end{array}
$$

where $a, b, c, d$ are complex parameters, $x$ and $y$ are complex variables, $y^{\prime}=d y / d x$. Equation (17) has three singular points $x=0, x=1$, and $x=\infty$. After multiplying by the common denominator, we obtain the equation as a differential sum. Its support and its polygon, in the case $a \neq 0$, $b \neq 0$, are shown in Fig. 11.

We found all formal asymptotic expansions (16) of solutions to equation (17) near its three singular points. They comprise 108 families. In particular, for $a=1 / 2$ and $c=0$, there is an expansion of the form

$$
y=-\frac{1}{\cos \left[\log \left(C_{1} x\right)\right]}+\sum_{\Re s \geqslant 1} c_{s} x^{s},
$$

where $C_{1}$ is an arbitrary constant, the coefficients $c_{s}$ are uniquely determined constants. Here

$$
\begin{aligned}
1 / \cos [\log (x)] & =2 /\left(x^{i}+x^{-i}\right)= \\
=2 x^{i} \sum_{k=0}^{\infty}\left(-x^{2 i}\right)^{k} & =2 x^{-i} \sum_{k=0}^{\infty}\left(-x^{-2 i}\right)^{k} .
\end{aligned}
$$

For $C_{1}=1$ and real $x>0$, solution (18) has infinitely many poles accumulating at the point $x=0$. We also found all expansions of solutions to equation (17) near its nonsingular points. They comprise 17 families [13].

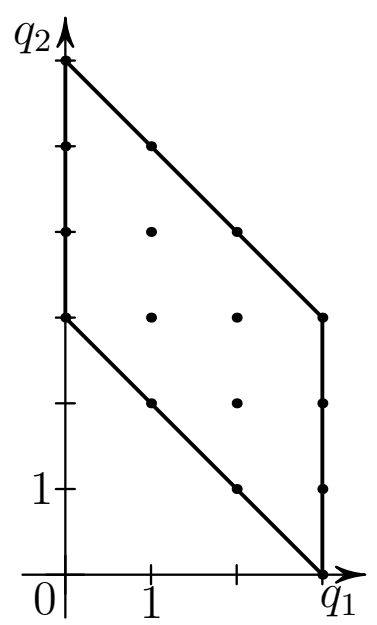

Figure 11. Support and Newton polygon for the sixth Painlevé equation (17)

That approach was applied to ODE systems [14-17].

\subsection{Applications}

1. Asymptotic forms and expansions of solutions to the Painlevé equations $[12,18,19]$.
2. Periodic motions of a satellite around its mass center moving along an elliptic orbit [20].

3. New properties of motion of a top (rigid body with a fixed point) [21].

4. Families of periodic solutions of the restricted threebody problem and distribution of asteroids [22,23].

5. Integrability of ODE systems [24].

6. Surface waves on water [3, Ch. 5].

\section{Ordinary Differential Equations. Differential Approach}

\subsection{Orders of solutions and their derivatives}

All solutions of the form (16) have the following property:

$$
p_{\omega}\left(y^{(k)}\right)=p_{\omega}(y)-k, \quad k=1,2, \ldots, n,
$$

where $n$ is the maximal order of derivative in the initial ODE, and

$$
p_{\omega}(\varphi)=\omega \limsup _{x^{\omega} \rightarrow \infty} \frac{\log |\varphi(x)|}{\omega \log |x|}
$$

along fixed $\arg x \in[0,2 \pi)$ is the order of the function $\varphi(x)$.

\subsection{Differential Approach [25-30]}

To each differential monomial $a(x, y)$ we put in correspondence the 3D point $\mathbf{Q}=\left(q_{1}, q_{2}, q_{3}\right)$, where $q_{1}$ and $q_{2}$ as before, but $q_{3}$ is the total order of derivatives in the monomial. We obtain the 3D support $\widetilde{\mathbf{S}}(f)$ of the initial ODE $f=0$ and polyhedron $\boldsymbol{\Gamma}(f)$ as convex hull of $\tilde{\mathbf{S}}(f)$. Using truncated equations, corresponding to its faces and edges, we can find their solutions in the form of elliptic or hyperelliptic functions $\varphi_{0}(x)$ and continue them into power-(hyper)elliptic expansion

$$
y=\varphi_{0}(x)+\sum_{l=1}^{\infty} \varphi_{l}(x) x^{-\omega l},
$$

where all $\varphi_{l}(x)$ are elliptic or hyperelliptic functions. This differential approach allows to find expansions (19) for solutions with property $p_{\omega}\left(y^{(k)}\right)=p_{\omega}(y)-\gamma_{\omega} k, k=$ $1,2, \ldots, n$, where $\gamma_{\omega} \neq 1$.

\section{Example 11 (cont. of Examples 6-10) [27]}

The 3D support $\tilde{\mathbf{S}}$ of the third Painleve equation (10) consists of 6 points:

$$
\begin{gathered}
\mathbf{Q}_{1}=(-1,2,2), \mathbf{Q}_{2}=(-1,2,1), \mathbf{Q}_{3}=(0,3,0), \\
\mathbf{Q}_{4}=(0,1,0), \mathbf{Q}_{5}=(1,4,0), \mathbf{Q}_{6}=(1,0,0) .
\end{gathered}
$$

Their convex hull $\Gamma$ is a pentahedron (Fig. 12). It has 2D upper face $\boldsymbol{\Gamma}_{1}^{(2)}$ with external normal $N_{1}=(1,0,1)$ containing 3 points of the support $\tilde{\mathbf{S}}$ : $\mathbf{Q}_{1}, \mathbf{Q}_{5}, \mathbf{Q}_{6}$. Corresponding truncated equation is

$$
\check{f}_{1}^{(2)} \stackrel{\text { def }}{=}-x y y^{\prime \prime}+x y^{\prime 2}+c x y^{4}+d x=0 .
$$

It has the first integral

$$
y^{\prime 2}=c y^{4}+C_{0} y^{2}-d \stackrel{\text { def }}{=} P(y),
$$




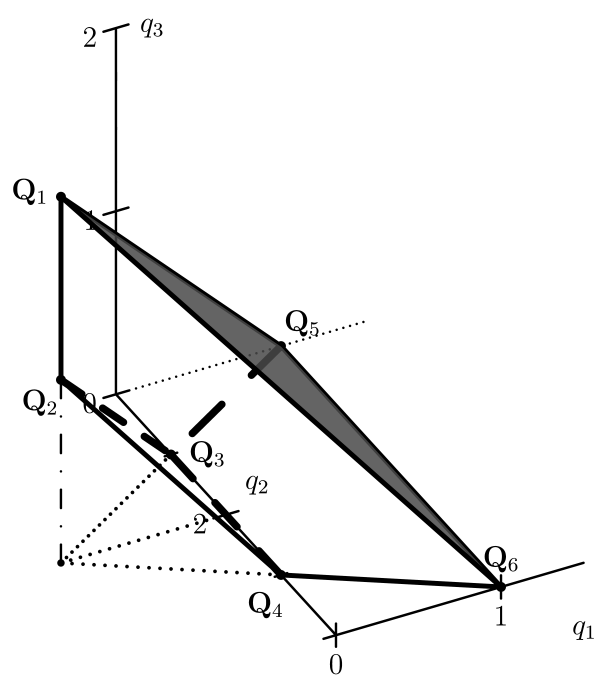

Figure 12. 3D support $\widetilde{\mathbf{S}}(f)$ and polyhedron $\boldsymbol{\Gamma}(f)$ of equation (10) with all $a, b, c, d \neq 0$. The grey face is $\boldsymbol{\Gamma}_{1}^{(2)}$. All dotted lines are in the plane $q_{1}$, $q_{2}$, they show projections of $\boldsymbol{\Gamma}(f)$ on the plane $\left(q_{1}, q_{2}\right)$.

where $C_{0} \in \mathbb{C}$ is an arbitrary constant. Discriminant of the polynomial $P(y)$ is $\Delta(P)=-c d\left(c d+C_{0}^{2} / 4\right)^{2}$. If $\Delta(P) \neq$ 0 , then solutions $y=\varphi_{0}(x)$ of the equation (20) are elliptic functions. So we can look for expansions (19), where all $\varphi_{l}(x)$ are regular functions of $\varphi_{0}(x)$. Here $\omega=1$ and $\gamma_{1}=$ 0 .

\section{Partial Differential Equations. Al- gebraic Approach}

\subsection{Theory [3]}

Let $X=\left(x_{1}, \ldots, x_{m}\right) \in \mathbb{C}^{m}$ be independent and $Y=$ $\left(y_{1}, \ldots, y_{n}\right) \in \mathbb{C}^{n}$ be dependent variables. Suppose $Z=$ $(X, Y) \in \mathbb{C}^{m+n}$. A differential monomial $a(Z)$ is the product of an ordinary monomial $c Z^{R}=c z_{1}^{r_{1}} \cdots z_{m+n}^{r_{m+n}}$, where $c=$ const $\in \mathbb{C}, R=\left(r_{1}, \ldots, r_{m+n}\right) \in \mathbb{R}^{m+n}$, and a finite number of derivatives of the form

$$
\begin{gathered}
\frac{\partial^{l} y_{j}}{\partial x_{1}^{l_{1}} \cdots \partial x_{m}^{l_{m}}} \stackrel{\text { def }}{=} \frac{\partial^{l} y_{j}}{\partial X^{L}}, \\
l_{j} \geqslant 0, \quad \sum_{j=1}^{m} l_{j}=l, \quad L=\left(l_{1}, \ldots, l_{m}\right) .
\end{gathered}
$$

A differential monomial $a(Z)$ corresponds to its vector power exponent $Q(a) \in \mathbb{R}^{m+n}$ formed by the following rules

$$
Q\left(c Z^{R}\right)=R, \quad Q\left(\partial^{l} y_{j} / \partial X^{L}\right)=\left(-L, E_{j}\right),
$$

where $E_{j}$ is unit vector. A product of monomials $a \cdot b$ corresponds to the sum of their vector power exponents: $Q(a b)=Q(a)+Q(b)$.

A differential sum is a sum of differential monomials $f(Z)=\sum a_{k}(Z)$. A set $\mathbf{S}(f)$ of vector power exponents $Q\left(a_{k}\right)$ is called the support of the sum $f(Z)$. The closure of the convex hull $\Gamma(f)$ of the support $\mathbf{S}(f)$ is called the polyhedron of the sum $f(Z)$.

Consider a system of equations

$$
f_{i}(X, Y)=0, \quad i=1, \ldots, n
$$

where $f_{i}$ are differential sums. Each equation $f_{i}=0$ corresponds to: its support $\mathbf{S}\left(f_{i}\right)$, its polyhedron $\Gamma\left(f_{i}\right)$ with the set of faces $\Gamma_{i j}^{\left(d_{i}\right)}$ in the main space $\mathbb{R}^{m+n}$, the set of normal cones $\mathbf{U}_{i j}^{\left(d_{i}\right)}$ to faces $\Gamma_{i j}^{\left(d_{i}\right)}$ in the dual space $\mathbb{R}_{*}^{m+n}$, and the set of truncated equations

$$
\hat{f}_{i j}^{\left(d_{i}\right)}(X, Y)=0 .
$$

The set of truncated equations

$$
\hat{f}_{i j_{i}}^{\left(d_{i}\right)}(X, Y)=0, \quad i=1, \ldots, n
$$

is the truncated system if the intersection of corresponding normal cones

$$
\mathbf{U}_{1 j_{1}}^{\left(d_{1}\right)} \cap \cdots \cap \mathbf{U}_{n j_{n}}^{\left(d_{n}\right)}
$$

is not empty. A solution $y_{i}=\varphi_{i}(X), i=1, \ldots, n$ to system (21) is associated to its normal cone $\mathbf{u} \subset \mathbb{R}^{m+n}$. If the normal cone $\mathbf{u}$ intersects with cone (23), then the asymptotic form $y_{i}=\hat{\varphi}_{i}(X), i=1, \ldots, n$ of this solution satisfies truncated system (22), which is quasi-homogeneous.

\subsection{Applications. Boundary layer on a needle [31]}

The theory of the boundary layer on a plate for a stream of viscous incompressible fluid was developed by Prandtl (1904) [32] and Blasius (1908) [33]. However a similar theory for the boundary layer on a needle was not known until recently, since no-slip conditions on the needle correspond to a more strong singularity as for the plate. This theory was developed with the help of Power Geometry (2004).

Let $x$ be an axis in three-dimensional real space, $r$ be the distance from the axis, and semi-infinite needle be placed on the half-axis $x \geqslant 0, r=0$. We studied stationary axisymmetric flows of viscous fluid which had constant velocity at $x=-\infty$ parallel to the axis $x$, and which satisfied noslip conditions on the needle (Fig. 13). We considered two cases: (1) incompressible fluid and (2) compressible heatconducting gas.

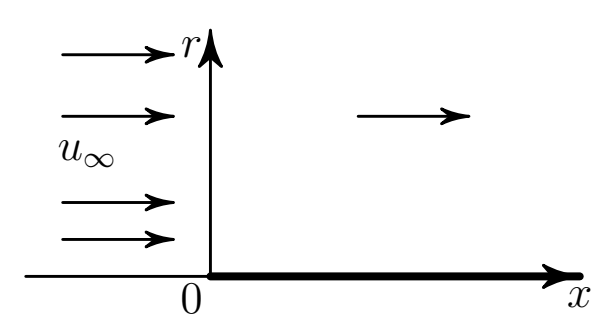

Figure 13. The needle and flow along it.

First case: incompressible fluid. For it, the NavierStokes equations in independent variables $x, r$ are equivalent to the system of two equations for the stream function $\psi$ and the pressure $p$

$$
\begin{aligned}
g_{1} & \stackrel{\text { def }}{=}-\frac{1}{r} \frac{\partial \psi}{\partial x} \frac{\partial}{\partial r}\left(\frac{1}{r} \frac{\partial \psi}{\partial r}\right)+\frac{1}{r} \frac{\partial \psi}{\partial r} \frac{\partial}{\partial x}\left(\frac{1}{r} \frac{\partial \psi}{\partial r}\right)+\frac{1}{\rho} \frac{\partial p}{\partial x}- \\
& -\nu\left(\frac{1}{r} \frac{\partial}{\partial r}\left(r \frac{\partial}{\partial r}\left(\frac{1}{r} \frac{\partial \psi}{\partial r}\right)\right)+\frac{\partial^{2}}{\partial x^{2}}\left(\frac{1}{r} \frac{\partial \psi}{\partial r}\right)\right)=0, \\
g_{2} & \stackrel{\text { def }}{=} \frac{1}{r} \frac{\partial \psi}{\partial x} \frac{\partial}{\partial r}\left(\frac{1}{r} \frac{\partial \psi}{\partial x}\right)-\frac{1}{r} \frac{\partial \psi}{\partial r} \frac{\partial}{\partial x}\left(\frac{1}{r} \frac{\partial \psi}{\partial x}\right)+\frac{1}{\rho} \frac{\partial p}{\partial r}+ \\
& +\nu\left(\frac{\partial}{\partial r}\left(\frac{1}{r} \frac{\partial^{2} \psi}{\partial x \partial r}\right)+\frac{\partial^{2}}{\partial x^{2}}\left(\frac{1}{r} \frac{\partial \psi}{\partial x}\right)\right)=0,
\end{aligned}
$$


where $\rho, \nu=$ const, with the boundary conditions

$$
\begin{aligned}
\psi & =\psi_{0} r^{2} \text { for } x=-\infty, \psi_{0}=\text { const } \\
\partial \psi / \partial x & =\partial \psi / \partial r=\partial^{2} \psi / \partial x \partial r=\partial^{2} \psi / \partial r^{2}=0
\end{aligned}
$$

for $x \geqslant 0, r=0$. Here $m=n=2$ and $m+n=4$.

Hence the supports of equations (24) must be considered in $\mathbb{R}^{4}$. It turned out that polyhedra $\Gamma\left(g_{1}\right)$ and $\Gamma\left(g_{2}\right)$ of equations (24) are three-dimensional tetrahedra, which can be moved by translation in one linear three-dimensional subspace, that simplified the isolation of the truncated systems. An analysis of truncated systems and of the results of their matching revealed that system (24) had no solution with $p \geqslant 0$ satisfying both boundary conditions (25), (26).

Second case: compressible heat-conducting gas. For this case, the Navier-Stokes equations in independent variables $x, r$ are equivalent to the system of three equations for the stream function $\psi$, the density $\rho$, and the enthalpy $h$ (an analog of the temperature)

$$
\begin{aligned}
& f_{1} \stackrel{\text { def }}{=}-\frac{1}{r} \frac{\partial \psi}{\partial x} \frac{\partial}{\partial r}\left(\frac{1}{\rho r} \frac{\partial \psi}{\partial x}\right) \\
& +\frac{1}{r} \frac{\partial \psi}{\partial r} \frac{\partial}{\partial x}\left(\frac{1}{\rho r} \frac{\partial \psi}{\partial x}\right)-A \frac{\partial}{\partial r}(\rho h) \\
& +\frac{2}{3} C^{N} \frac{\partial}{\partial r}\left(\frac{h^{N}}{r} \frac{\partial}{\partial r}\left(\frac{1}{\rho} \frac{\partial \psi}{\partial x}\right)\right) \\
& -\frac{2}{3} C^{N} \frac{\partial}{\partial r}\left(\frac{h^{N}}{r} \frac{\partial}{\partial x}\left(\frac{1}{\rho} \frac{\partial \psi}{\partial r}\right)\right) \\
& -\frac{2 C^{N}}{r} \frac{\partial}{\partial r}\left(h^{N} r \frac{\partial}{\partial r}\left(\frac{1}{\rho r} \frac{\partial \psi}{\partial x}\right)\right) \\
& +C^{N} \frac{\partial}{\partial x}\left(h^{N} \frac{\partial}{\partial r}\left(\frac{1}{\rho r} \frac{\partial \psi}{\partial r}\right)\right) \\
& -C^{N} \frac{\partial}{\partial x}\left(h^{N} \frac{\partial}{\partial x}\left(\frac{1}{\rho r} \frac{\partial \psi}{\partial x}\right)\right) \\
& +\frac{2 C^{N} h^{N}}{\rho r^{3}} \frac{\partial \psi}{\partial x}=0, \\
& f_{2} \stackrel{\text { def }}{=} \frac{1}{r} \frac{\partial \psi}{\partial x} \frac{\partial}{\partial r}\left(\frac{1}{\rho r} \frac{\partial \psi}{\partial r}\right) \\
& -\frac{1}{r} \frac{\partial \psi}{\partial r} \frac{\partial}{\partial x}\left(\frac{1}{\rho r} \frac{\partial \psi}{\partial r}\right)-A \frac{\partial}{\partial x}(\rho h) \\
& +\frac{2}{3} C^{N} \frac{\partial}{\partial x}\left(\frac{h^{N}}{r} \frac{\partial}{\partial r}\left(\frac{1}{\rho} \frac{\partial \psi}{\partial x}\right)\right) \\
& -\frac{2}{3} C^{N} \frac{\partial}{\partial x}\left(\frac{h^{N}}{r} \frac{\partial}{\partial x}\left(\frac{1}{\rho} \frac{\partial \psi}{\partial r}\right)\right) \\
& +\frac{C^{N}}{r} \frac{\partial}{\partial r}\left(h^{N} r \frac{\partial}{\partial r}\left(\frac{1}{\rho r} \frac{\partial \psi}{\partial r}\right)\right) \\
& -\frac{C^{N}}{r} \frac{\partial}{\partial r}\left(h^{N} r \frac{\partial}{\partial x}\left(\frac{1}{\rho r} \frac{\partial \psi}{\partial x}\right)\right) \\
& +2 C^{N} \frac{\partial}{\partial x}\left(h^{N} \frac{\partial}{\partial x}\left(\frac{1}{\rho r} \frac{\partial \psi}{\partial r}\right)\right)=0,
\end{aligned}
$$

$$
\begin{aligned}
f_{3} & \stackrel{\text { def }}{=} \frac{1}{r} \frac{\partial \psi}{\partial x} \frac{\partial h}{\partial r}-\frac{1}{r} \frac{\partial \psi}{\partial r} \frac{\partial h}{\partial x}-\frac{A}{\rho r} \frac{\partial \psi}{\partial x} \frac{\partial(\rho h)}{\partial r} \\
& +\frac{A}{\rho r} \frac{\partial \psi}{\partial r} \frac{\partial(\rho h)}{\partial x}+2 C^{N} h^{N}\left(\frac{\partial}{\partial r}\left(\frac{1}{\rho r} \frac{\partial \psi}{\partial x}\right)\right)^{2} \\
& +2 C^{N} h^{N}\left(\frac{1}{r^{2} \rho} \frac{\partial \psi}{\partial x}\right)^{2}+2 C^{N} h^{N}\left(\frac{\partial}{\partial x}\left(\frac{1}{\rho r} \frac{\partial \psi}{\partial r}\right)\right)^{2} \\
& +C^{N} h^{N}\left(\frac{\partial}{\partial x}\left(\frac{1}{\rho r} \frac{\partial \psi}{\partial x}\right)\right)^{2} \\
& -C^{N} h^{N} \frac{\partial}{\partial x}\left(\frac{1}{\rho r} \frac{\partial \psi}{\partial x}\right) \frac{\partial}{\partial r}\left(\frac{1}{\rho r} \frac{\partial \psi}{\partial r}\right) \\
& +C^{N} h^{N}\left(\frac{\partial}{\partial r}\left(\frac{1}{\rho r} \frac{\partial \psi}{\partial r}\right)\right)^{2} \\
& -\frac{2}{3} C^{N} h^{N}\left(\frac{1}{r} \frac{\partial}{\partial r}\left(\frac{1}{\rho} \frac{\partial \psi}{\partial x}\right)\right)^{2} \\
& +\frac{4 C^{N} h^{N}}{3 r} \frac{\partial}{\partial r}\left(\frac{1}{\rho} \frac{\partial \psi}{\partial x}\right) \frac{\partial}{\partial x}\left(\frac{1}{\rho r} \frac{\partial \psi}{\partial r}\right) \\
& -\frac{2}{3} C^{N} h^{N}\left(\frac{\partial}{\partial x}\left(\frac{1}{\rho r} \frac{\partial \psi}{\partial r}\right)\right)^{2} \\
+ & \frac{C^{N}}{\sigma r} \frac{\partial}{\partial r}\left(r h^{N} \frac{\partial h}{\partial r}\right)+\frac{C^{N}}{\sigma} \frac{\partial}{\partial x}\left(h^{N} \frac{\partial h}{\partial x}\right)=0 \\
&
\end{aligned}
$$

where parameters $A, C, \sigma>0$ and $N \in[0,1]$, with the boundary conditions

$$
\begin{aligned}
& \psi=\psi_{0} r^{2}, \rho=\rho_{0}, h=h_{0} \text { for } \\
& x=-\infty, \psi_{0}, \rho_{0}, h_{0}=\mathrm{const}
\end{aligned}
$$

and (26). Here $m=2, n=3$, and $m+n=5$. In the space $\mathbb{R}^{5}$, all polyhedrons $\Gamma\left(f_{1}\right), \Gamma\left(f_{2}\right), \Gamma\left(f_{3}\right)$ of equations (29) are three-dimensional, and they can be translated into one linear 3D subspace. In coordinates $\tilde{Q}^{\prime}=\left(\tilde{q}_{1}^{\prime}, \tilde{q}_{2}^{\prime}, \tilde{q}_{3}^{\prime}\right)$ of this threedimensional space, the supports and polyhedra are shown in Figures 14-16.

The supports of sums $f_{1}, f_{2}$ and $f_{3}$ are following:

$$
\begin{gathered}
\mathbf{S}\left(f_{1}\right)=\left\{\tilde{Q}_{0}^{\prime}=0, \tilde{Q}_{1}^{\prime}=(1,0,0), \tilde{Q}_{2}^{\prime}=(0,1,0),\right. \\
\left.\tilde{Q}_{3}^{\prime}=(0,0,1)\right\}, \\
\mathbf{S}\left(f_{2}\right)=\left\{\tilde{Q}_{0}^{\prime}=0, \tilde{Q}_{1}^{\prime}=(1,-1,1), \tilde{Q}_{2}^{\prime}=(0,1,0),\right. \\
\left.\tilde{Q}_{3}^{\prime}=(0,0,1)\right\}, \\
\mathbf{S}\left(f_{3}\right)=\left\{\tilde{Q}_{0}^{\prime}=0, \tilde{Q}_{1}^{\prime}=(0,1,0), \tilde{Q}_{2}^{\prime}=(-1,2,-1),\right. \\
\left.\tilde{Q}_{3}^{\prime}=(0,0,1), \tilde{Q}_{4}^{\prime}=(-1,0,1), \tilde{Q}_{5}^{\prime}=(-1,1,0)\right\} .
\end{gathered}
$$

The truncated system corresponding to the boundary layer on the needle corresponds to the vertex $\tilde{Q}_{1}^{\prime}$, to faces $\left[\tilde{Q}_{0}^{\prime}, \tilde{Q}_{1}^{\prime}, \tilde{Q}_{2}^{\prime}\right]$ and $\left[\tilde{Q}_{0}^{\prime}, \tilde{Q}_{1}^{\prime}, \tilde{Q}_{2}^{\prime}\right]$ of polyhedrons $\Gamma\left(f_{1}\right), \Gamma\left(f_{2}\right)$ and $\Gamma\left(f_{3}\right)$ respectively. In Figs. 14-16 they are distinguished. 


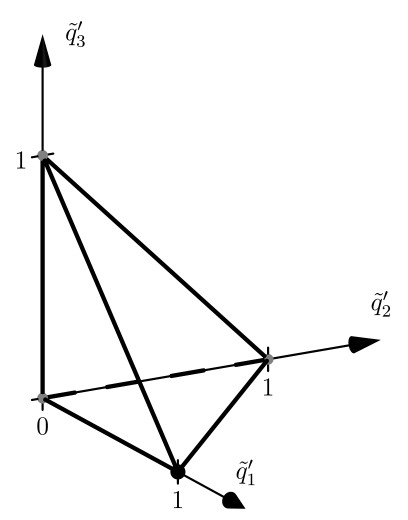

Figure 14. The Newton polyhedron of $f_{1}$ in (29)

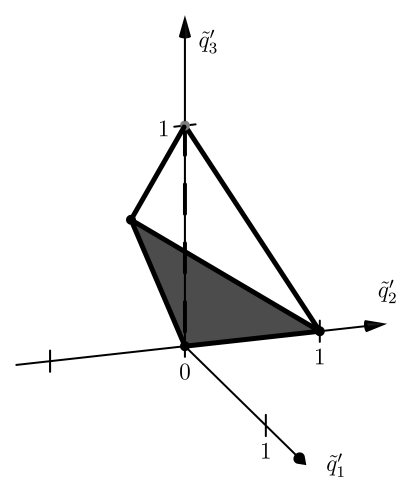

Figure 15. The Newton polyhedron of $f_{2}$ in (29)

Thus, the truncated system is

$$
\begin{aligned}
\hat{f}_{12}^{(0)} & \stackrel{\text { def }}{=}-A \partial(\rho h) / \partial r=0, \\
\hat{f}_{22}^{(2)} & \stackrel{\text { def }}{=} \frac{1}{r} \frac{\partial \psi}{\partial x} \frac{\partial}{\partial r}\left(\frac{1}{\rho r} \frac{\partial \psi}{\partial r}\right)-\frac{1}{r} \frac{\partial \psi}{\partial r} \frac{\partial}{\partial x}\left(\frac{1}{\rho r} \frac{\partial \psi}{\partial r}\right)- \\
& -A \frac{\partial}{\partial x}(\rho h)+\frac{C^{N}}{r} \frac{\partial}{\partial r}\left(h^{N} r \frac{\partial}{\partial r}\left(\frac{1}{\rho r} \frac{\partial \psi}{\partial r}\right)\right)=0, \\
\hat{f}_{32}(2) & \stackrel{\text { def }}{=} \frac{1}{r} \frac{\partial \psi}{\partial x} \frac{\partial h}{\partial r}-\frac{1}{r} \frac{\partial \psi}{\partial r} \frac{\partial h}{\partial x}-\frac{A}{\rho r} \frac{\partial \psi}{\partial x} \frac{\partial(\rho h)}{\partial r}+ \\
& +\frac{A}{\rho r} \frac{\partial \psi}{\partial r} \frac{\partial(\rho h)}{\partial x}+C^{N} h^{N}\left(\frac{\partial}{\partial r}\left(\frac{1}{\rho r} \frac{\partial \psi}{\partial r}\right)\right)^{2}+ \\
& +\frac{C^{N}}{\sigma r} \frac{\partial}{\partial r}\left(r h^{N} \frac{\partial h}{\partial r}\right)=0,
\end{aligned}
$$

with the boundary conditions $\psi=\psi_{0} r^{2}, \rho=\rho_{0}, h=h_{0}$; $\psi_{0}, \rho_{0}, h_{0}=$ const, for $r \rightarrow \infty$.

An analysis of solutions to the latter problem (31) by methods of Power Geometry revealed that for $N \in(0,1)$ it has

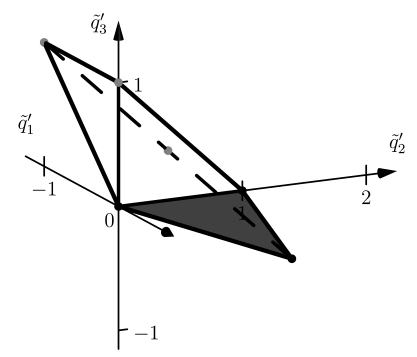

Figure 16. The Newton polyhedron of $f_{3}$ in (29) solutions of the form

$\psi \sim c_{1} r^{2}|\log \xi|^{-1 / N}, \rho \sim c_{2}|\log \xi|^{-1 / N}, h \sim c_{3}|\log \xi|^{1 / N}$,

where $\xi=r^{2} / x \rightarrow 0$ and $c_{1}, c_{2}, c_{3}$ are arbitrary real constants. Thus, for $N \in(0,1)$, in the boundary layer $r^{2} / x<$ const, as $x \rightarrow+\infty$ and $\xi=r^{2} / x \rightarrow 0$, we obtained the asymptotic form of the flow (32), i.e. near the needle, the density $\rho$ tends to zero, and the temperature $h$ increases to infinity as the distance $x$ to the initial point of the needle tends to $+\infty$.

\subsection{Other applications of Power Geometry}

Evolution of the turbulent flow [34,35] and Thermodynamics [36] and power-elliptic expansions of solutions to Painlevé equations [37].

\section{Connection with Idempotent Math- ematics}

For polynomial $f(X)$, let us define the function

$$
\hat{f}(S)=\lim _{h \rightarrow+0} h \log |f(\exp (S / h))|
$$

and its subdifferential

$$
\partial \hat{f}=\left\{Q \in \mathbb{R}^{n}:\langle Q, S\rangle \leqslant \hat{f}(S) \text { for all } S \in \mathbb{R}_{*}^{n}\right\} .
$$

Theorem 6 ( [38]) If $f(X)$ is a polynomial, then the subdifferential $\partial \hat{f}$ of $f(X)$ at the origin coincides with the Newton polyhedron $\Gamma(f)$.

V. P. Maslov and his colleagues developed Idempotent Analysis [38, 39]. However, as a method of finding leading terms in nonlinear problems, it is too complicated. Theorem 6 shows that in algebraic problems Idempotent Analysis gives the Newton polyhedron. This observation can be generalized to other classes of problems, but if we just begin with appropriate generalization of the Newton polyhedron (or Power Geometry), then we do not really need Idempotent Analysis (see Sections 4 and 5). Indeed, Idempotent Analysis [39] is useful in problems with "bad" solutions (for instance, discontinuous or non-smooth).

\section{REFERENCES}

[1] Bruno, A.D.: The asymptotic behavior of solutions of nonlinear systems of differential equations. DAN SSSR 143:4, 763-766 (1962) (in Russian). English translation: Soviet Math. Dokl. 3 464-467 (1962)

[2] Bruno, A.D.: Local Methods in Nonlinear Differential Equations. Moscow, Nauka (1979) (in Russian). English translation: Springer-Verlag, Berlin-New York (1989)

[3] Bruno, A.D.: Power Geometry in Algebraic and Differential Equations. Moscow, Fizmatlit (1998) (in Russian). English translation: Elsevier, Amsterdam (2000) 
[4] Bruno, A.D., Batkhin, A.B.: Asymptotic solution of an algebraic equation. DAN 440:3 , 295300 (2011). English translation: Doklady Mathematics 84:2, 634-639 (2011) http://dx.doi.org/10. 1134/S1064562411060160.

[5] Newton, I.: The Method of Fluxions and Infinite Series with its Applications to the Geometry of Curve Lines. New York; London, Johnson Reprint Corp. (1964)

[6] Briot, C., Bouquet, T.: Recherches sur les propriétés des équations differentielles. J. Ecole Polytechn. Paris, 21, No 36 (1856).

[7] Mikhailov, V.P.: On the first boundary value problem for some semi-bounded operators. Dokl. Akad. Nauk SSSR, 151, 282-285 (1963) (in Russian). English translation: Sov. Math. Dokl. 4, 997-1001 (1963)

[8] Bruno, A.D.: On geometric methods in works by V.I. Arnold and V.V. Kozlov. Preprint ArXiv: 1401.6320v1.

[9] Bruno, A.D., Batkhin, A.B.: Resolution of algebraic singularity by algorithms of Power Geometry. Programmirovanie. 38:2, 12-30 (2012) (in Russian). English translation: Programming and Computer Software. 38:2, 57-72 (2012) http://dx.doi.org/10. 1134/S036176881202003X.

[10] Batkhin, A.B., Bruno, A.D., Varin, V.P.: Sets of stability of multiparameter Hamiltonian systems. PMM. 76:1, 80-133 (2012) (in Russian). English translation: J. Appl. Math. Mech. 76:1, 56-92 (2012) http://dx.doi.org/10.1016/j.jappmathmech . 2012.03 .006 .

[11] Bruno, A.D.: Asymptotics and expansions of solutions to an ordinary differential equation. UMN. 59:3, 3180 (2004) (in Russian). English translation: Russian Mathem. Surveys. 59:3, 429-480 (2004) http://dx . doi .org/10.1070/RM2004v059n03ABEH000736.

[12] Bruno, A.D., Goryuchkina, I.V.: Asymptotic expansions of solutions of the sixth Painlevé equation. Trudy Mosk. Mat.Obs. 71, 6-118 (2010) (in Russian). English translation: Transactions of Moscow Math. Soc. 71, 1-104 (2010) http://dx.doi.org/10.1090/ s0077-1554-2010-00186-0.

[13] Bruno, A.D., Goryuchkina, I.V.: All expansions of solutions to the sixth Painlevé equation near its nonsingular point. Doklady Akademii Nauk. 426:5, 586591 (2009) (in Russian). English translation: Doklady Mathematics. 79:3, 397-402 (2009) http://dx.doi. org/10.1134/S1064562409030260.

[14] Bruno, A.D.: Power asymptotics of solutions to an ODE system. DAN. 410:5, 583-586 (2006) (in Russian). English translation: Doklady Mathematics. 74:2, 712-715 (2006) http://dx.doi.org/10. 1134/S1064562406050243.

[15] Bruno, A.D.: Power-logarithmic expansions of solutions to a system of ordinary differential equations. DAN. 419:3, 298-302 (2008) (in Russian). English translation: Doklady
Mathematics. 77:2, 215-218 (2008) http: //dx.doi.org/10.1134/S1064562408020154

[16] Bruno, A.D.: Nonpower asymptotic forms of solutions to a system of ordinary differential equations. DAN. 420:1, 7-10 (2008) (in Russian). English translation: Doklady Mathematics. 77:3, 325-328 (2008) http: //dx.doi.org/10.1134/S1064562408030010.

[17] Bruno, A.D.: Complicated expansions of solutions to a system of ordinary differential equations. DAN. 421:1, 7-10 (2008) (in Russian). English translation: Doklady Mathematics. 78:1, 477-480 (2008) http://dx.doi. org/10.1134/S1064562408040017.

[18] Bruno, A.D., Parusnikova, A.V.: Local expansions of solutions to the fifth Painlevé equation. 438:4, 439443 (2011) (in Russian). English translation: Doklady Mathematics. 83:3, 348-352 (2011) http://dx.doi. org/10.1134/S1064562411030276.

[19] Bruno, A.D., Parusnikova, A.V.: Expansions of solutions to the fifth Painlevé equation near its nonsingular point. DAN, 442:5, 583-588 (2012) (in Russian). English translation: Doklady Mathematics. 85:1, 87-92 (2012) http://dx.doi.org/10.1134/ S1064562412010292

[20] Bruno, A.D.: Families of periodic solutions to the Beletsky equation. Kosmicheskie Issledovanija. 40:3, 295-316 (2002) (in Russian). English translation: Cosmic Research. 40:3, 274-295 (2002)

[21] Bruno, A.D.: Analysis of the Euler-Poisson equations by methods of Power Geometry and Normal Form. PMM. 71:2, 192-227 (2007) (in Russian). English translation: J. Appl. Math. Mech. 71:2, 168-199 (2007) http://dx.doi.org/10.1016/j. jappmathmech.2007.06.002.

[22] Bruno, A.D., Varin, V.P.: Periodic solutions of the restricted three-body problem for small mass ratio. PMM .71:6, 1034-1066 (2007) (in Russian). English translation: J. Appl. Math. Mech. 71:6, 933-960 (2007) http://dx.doi.org/10.1016/j. jappmathmech.2007.12.012

[23] Bruno, A.D., Varin, V.P.: On asteroid distribution. Astronomicheskii Vestnik. 45:4, 334-340 (2011) (in Russian). English translation: Solar System Research. 45:4, 323-329 (2011) http://dx.doi.org/ 10.1134/S0038094611040010.

[24] Bruno, A.D., Edneral, V.F.: Algorithmic analysis of local integrability. DAN. 424:3, 299-303 (2009) (in Russian). English translation: Doklady Mathematics. 79:1, 48-52 (2009) http://dx.doi.org/10.1134/ S1064562409010141

[25] Bruno, A.D.: Space Power Geometry for an ODE and Painleve equations.In: International Conference "Painleve Equations and Related Topics". pp. 36-41. St. Petersburg, June, (2011)

[26] Bruno, A.D.: Power-exponential expansions of solutions to an ordinary differential equation. DAN. 444:2, 
137-142 (2012) (in Russian). English translation: Doklady Mathematics. 85:3, 336-340 (2012) http: //dx. doi.org/10.1134/S106456241203009X.

[27] Bruno, A.D.: Space Power Geometry for an ODE and $P_{1}-P_{4}, P_{6}$. In: Bruno, A.D., Batkhin, A.B. (eds.) Painlevé Equations and Related Topics, pp. 41-51. De Gruyter, Berlin/Boston (2012)

[28] Bruno, A.D., Parusnikova, A.V.: Elliptic and periodic asymptotic forms of solutions to $P_{5}$. In: Bruno, A.D., Batkhin, A.B. (eds.) Painlevé Equations and Related Topics (Eds. A.D. Bruno and A.B. Batkhin), pp. 53-65. De Gruyter, Berlin/Boston (2012)

[29] Bruno, A.D.: Regular asymptotic expansions of solutions to one ODE and $P_{1}-P_{5}$. Bruno, A.D., Batkhin, A.B. (eds.) Painlevé Equations and Related Topics (Eds. A.D. Bruno and A.B. Batkhin), pp. 67-82. De Gruyter, Berlin/Boston (2012)

[30] Bruno, A.D.: Power-elliptic expansions of solutions to an ODE. Zhurnal Vychisl. Matem. i Matem. Fiziki. 52:12, 2206-2218 (2012) (in Russian). English translation: Comp. Mathem. Math. Phys. 52:12, 1650-1661 (2012) http://dx.doi.org/10.1134/ S0965542512120056.

[31] Bruno, A.D., Shadrina, T.V.: Axisymmetric boundary layer on a needle. Trudy Mosk. Mat. Obsch. 68. 224-287 (2007) (in Russian). English translation: Transactions of Moscow Math. Soc. 68, 201-259 (2007) http://dx.doi.org/10.1090/ s0077-1554-07-00165-3.

[32] Prandtl, L.: Über Flüssigkeitsbewegung bei sehr kleiner Reibung. In: Verhandl. III Kongr., Heidelberg (1904)
[33] Blasius, H.: Grenzschichten in Flüssigkeiten mit kleiner Reibung. Zeit. für Math. und Phys. 56, 1-37 (1908)

[34] Bruno, A.D.: Power geometry in nonlinear partial differential equations. Ukrainean Mathem. Bulletin. 5:1, 32-45 (2008)

[35] Bruno, A.D.: Power geometry in differential equations. "Contemporary Problems of Mathematics and Mechanics". Mathematics. Dynamical Systems. MGU. Moscow, 4:2, 24-54 (2009) (in Russian)

[36] Bruno, A.D.: Self-similar solutions and Power Geometry. Uspekhi Mat. Nauk. 55:1, 3-44 (2000) (in Russian). English translation: Russian Math. Surveys, 55:1, 1-42 (2000) http://dx.doi.org/10.1070/ RM2000v055n01ABEH000248.

[37] Bruno, A.D.: Power geometry and elliptic expansions of solutions to the Painlevé equations. International Journal of Differential Equations, V. 2015. Article ID 340715, 13 p http://dx.doi.org/10.1155/2015/ 340715 .

[38] Litvinov, G.L., Shpiz, G.B.: The dequantization transform and generalized Newton polytopes. In: Litvinov, G.L., Maslov, V.P. (eds.) Idempotent Mathematics and Mathematical Physics. pp. 99-104. Contemporary Mathematics, 377, AMS, Providence, RI (2005)

[39] Maslov, V.P., Kolokoltsov, V.N.: Idempotent Analysis and Its Applications to Optimal Control Theory. Moscow, Nauka (1994) (in Russian) 